\title{
HyHOPE: Fast fault simulator with efficient simulation of hypertrophic faults
}

\author{
C.-P. Kung \\ C.-S. Lin
}

Indexing term: Circuit theory and design

\begin{abstract}
In sequential circuit fault simulation, hypertrophic faults, which result from a lengthened initialisation sequence in faulty circuits, usually produce a large number of fault events during simulation and require excessive gate evaluations. These faults degrade the performance of fault simulators attempting to simulate them exactly. In the paper, an exact simulation algorithm is developed to identify hypertrophic faults and to minimise their effects during fault simulation. The simulator HyHOPE, based on this algorithm, shows that the average speed-up ratio over HOPE for test vectors generated by STG3 is 1.65 . Furthermore, the results indicate that the performance of HyHOPE is close to the approximate simulator, in which hypertrophic faults are simply dropped when they become potentially detected.
\end{abstract}

\section{Introduction}

Fault simulation has played a major role in VLSI testing. Its applications range from grading the quality of test sets to incorporating ATPG in test generation [17-20]. As the size of VLSI circuits grows larger, efficient fault simulation algorithms have been developed to meet the challenge [1-16]. These algorithms are highly refined for their domains of application. In this paper, we are mainly concerned with fault simulation algorithms for single stuck-at faults of synchronous sequential circuits.

One popular simulation algorithm is the concurrent fault simulation algorithm [13]. In concurrent fault simulation, a descriptor for a faulty circuit is present in the fault list of a gate only if the faulty-circuit status is different from that of the good circuit. A fault copy for a gate is simulated only when the fault is in the fault list, and a logic event or a fault event of this gate input occurs. The major advantage of concurrent fault simulation is its versatility. It is applicable to mixed-level circuits, various numbers of signal values and any gate delay model [14]. However, it requires large memory, and its performance is slower than the other fault simulation algorithms to be discussed below.

Most recently developed fault simulation algorithms are based on the single-fault propagation algorithm

(C) IEE, 1995

Paper 1623G (E10), first received 18th November 1993 and in revised form 26th September 1994

The authors are with the Department of Electrical Engineering, Nationa) Taiwan University, Taipei, Taiwan, Republic of China
ROOFS [3]. In single-fault propagation [1], faults are simulated one at a time, and only the difference between a faulty circuit and the good circuit is simulated. Furthermore, only a linked list is maintained for each faulty circuit to record its difference in status from the good circuit. Within the circuit, one set of fault-status words is maintained and shared by all faults. Hence, the memory requirement is much lower than for concurrent fault simulation. To efficiently simulate one fault after another without restoring status, for each simulated fault of each time frame, ROOFS assigns a unique simulation ID to indicate whether the fault-status word belongs to the currently simulated faulty circuit. Using this technique, the number of gate evaluations is significantly reduced.

Based on ROOFS, various parallelisation techniques have been proposed to improve performance further. Two fault simulators endeavour to parallelise the test vectors: PSF [6] and PARIS [7]. The major difference between PSF and PARIS lies in their way of grouping test vectors into a packet, or computer word. In PSF, the test sequence is partitioned into consecutive subsequences, whereas a packet in PARIS represents consecutive test vectors. Owing to a different test sequence arrangement, PSF performs multiple-pass simulation, and PARIS performs multiple iterations for a packet to obtain accurate results. Both PSF and PARIS can achieve significant speed-up over ROOFS, although their performance is somewhat correlated with the circuit types.

PROOFS [3, 4] is the parallel-fault enhancement of ROOFS. In PROOFS, a packet of 32 active faults is injected and simulated together using bit parallelism of computer word operation. In addition to simulating faults in parallel, PROOFS dynamically arranges the faults in a packet such that only the active faults are simulated. An even more efficient parallel-fault simulator, HOPE, is proposed in Reference 5. In HOPE, an active fault at a time frame is categorised as a single-event fault, if the fault effect originates only from the fault site, i.e. the present state of the faulty circuit is the same as that of the good circuit. The single-event faults are then simulated with sophisticated techniques similar to those found in combinational fault simulation. The results in Reference 5 show that, on average, $67 \%$ of faults are screened out, and a speed-up of 2 is obtained, compared with PROOFS.

Despite these sophisticated techniques, the efficiency of the fault simulated algorithms above depends on the

The authors would like to thank Dr. Dong Sam Ha for providing the source code of HOPE. 
degree of difference between a good circuit and its faulty versions, i.e. the fault effects. For most faults, the fault effects are few in number, and these simulators attain high efficiency by evaluating only a small number of gates for each fault. However, there are usually some faults in a circuit that take a longer sequence and are difficult to initialise. Hence, while a good circuit is initialised, these faults produce a great number of unknown values $(X s)$, which are evidently different from good circuit values. As a result, a simulator taking advantage of such differences ends up heavily loaded with a large number of gate evaluations for the hypertrophic faults Such faults are named hypertrophic faults in Reference 10. In HOPE, almost half the fault events are from such hypertrophic faults on ISCAS89 benchmark circuits, with test sequences generated by the reversed-time-approach test generator STG3 [18-20]. Its performance suffers greatly from these faults. This phenomenon has long been known, and the traditional way of dealing with hypertrophic faults is to drop these faults as potentially detected once the faulty $X$ s propagate to POs along with known good circuit values. Accuracy is thus sacrificed for higher simulation speed.

In this paper, we propose a novel and exact fault simulation algorithm to identify hypertrophic faults during fault simulation and to simulate efficiently hypertrophic faults for sequential circuits, such that their adverse effect on performance is minimised. Based on the simulation algorithm proposed, the reduction of gate evaluations resulting from hypertrophic faults is threefold:

(a) The algorithm performs gate evaluation only when there is a difference with the previous time frame rather than with the good circuit.

(b) Each fault is simulated in parallel with logic simulation.

(c) The faulty circuits of various hypertrophic faults are simulated in parallel for an even greater reduction.

From this algorithm, a fault simulator for efficient and exact hypertrophic fault simulation, HyHOPE, is implemented on the framework of HOPE. The experimental results show that HyHOPE reduces above $40 \%$ of gate evaluations for faulty-circuit simulation of HOPE, and the average speed-up ratio over HOPE for test vectors generated by STG3 is 1.65 . Furthermore, the results indicate that the performance of HyHOPE is close to an approximate version of HOPE, in which potentially detected faults are dropped.

\section{Hypertrophic faults}

In this Section, the behaviour of hypertrophic faults and their identification will be described. Furthermore, the key observation for reducing the hypertrophic fault effect will be discussed.

\subsection{Definition}

Hypertrophic faults [10] are faults that lengthen the initialisation of faulty circuits and, during this period, cause the status of many gates in the faulty circuits to remain unknown $(X s)$, when the good circuit has been initialised. In general, such hypertrophic faults in a circuit are few in number and are usually associated with control lines, such as faults on reset lines. However, their fault effects, or difference from a good circuit, spread widely at each time-frame and generate a great number of fault events and gate evaluations, such that the performance of fault simulators is significantly degraded. The degradation in performance stems from the fact that modern fault simulators count precisely on the small difference between the good circuit and its faulty versions. This difference is small for nonhypertrophic faults, which form the majority, and these fault simulators achieve good efficiency. The opposite characteristic of hypertrophic faults seriously affects the attainable efficiency of these simulators.

To handle hypertrophic faults, two approaches have been taken in the literature. One approach is to handle these faults implicitly, through either dynamic fault ordering [11] or dynamic fault grouping [12]. Both manipulate the ordering or group of faults during simulation to reduce the fault events. This approach is intuitive, but the improvement is not significant. In particular, the results of Reference 12 show that the number of fault events can only be reduced about $17 \%$ for two of the benchmark circuits and, for the other circuits, dynamic fault grouping gives an even smaller reduction.

Another approach is to relax the detection condition from sure detection to potential detection. A fault is surely detected if the fault produces a complementary value of the good circuit at primary outputs. A fault is potentially detected if there exists a primary output whose faulty-circuit value is unknown when the goodcircuit value is known. As most hypertrophic faults are also potentially detectable, if a fault is dropped when it is potentially detected, the simulation time will be significantly reduced. Once it has been dropped, a potentially detected fault's detectability remains uncertain, even though some faults still have the possibility of being surely detected in later time frames. Accuracy is thus traded for faster simulation time. The simulation result is approximate rather than exact.

We propose an exact fault simulator that minimises the simulation requirement for hypertrophic faults. First, the identification of hypertrophic faults will be described.

\subsection{Identification}

The first step when handling hypertrophic faults is to identify these faults during the simulation. There have been a few methods to identify a hypertrophic fault. It can be either simply a fault that has been potentially detected or, as in Mozart [10], a fault whose difference from the good circuit is comparable in size to the goodcircuit events. These methods are more suitable for approximate fault simulation, in which these identified faults are dropped.

To simulate hypertrophic faults exactly without incurring much overhead, these faults should be identified based on the estimation of $X s$ at the next time frame. The number of flip-flops at which the faulty-circuit values are unknown and different from those of the good circuit is a good estimate for this purpose. Since from more $X \mathbf{S}$ at FFs, it is more likely that the difference with good circuit at the next time frame will be larger. If such a fault count is larger than a predetermined threshold value, the fault is identified as hypertrophic. The lower the threshold value, the sooner a hypertrophic fault is identified. However, there is a risk that some faults will be incorrectly identified as hypertrophic. Hence, the threshold value is a trade-off between these two extremes. In our implementation, the threshold value is empirically determined as $5 \%$ of the number of flip-flops in a circuit. The overhead for identification is negligible compared with the entire simulation time.

\subsection{Key observation}

Once the hypertrophic faults have been identified, an effective simulation algorithm can be developed to simu- 
late them exactly. The simulation algorithm is based on the following observation: In event-driven simulation algorithms (logic or fault simulation) gate evaluation is required for a gate if any event occurs in the gate inputs. In other words, gate evaluation is required if the gate input status is different from the reference circuit status. The reference circuit can be the same circuit in the previous time frame, as in logic simulation, or the good circuit in the same time frame, as in ROOFS. Hence, a fault simulation algorithm is more efficient if it has fewer fault events. The following observation shows that hypertrophic fault events can be reduced if a proper reference circuit is chosen:

Key observation: A hypertrophic fault tends to have fewer events with respect to the same faulty circuit in the previous time frame than with respect to the good circuit. Furthermore, these events are highly correlated with the logic events of the good circuit. In other words,

$$
\left|E_{\boldsymbol{f}-\boldsymbol{g}}\right| \gg\left|E_{f}-E_{g}\right|
$$

where $E_{f-g}$ are the events corresponding to the difference between the faulty circuit and the good circuit in the current time frame; $E_{f}$ are the events corresponding to the difference in the faulty circuit between the current time frame and the previous time frame, $E_{g}$ are the logic events corresponding to the difference in the good circuit between the current time frame and the previous time frame; and the minus sign in $E_{f}-E_{g}$ is the set difference.

The validity of the observation can be demonstrated in Table 1 and will be further discussed in the gate evalu-

Table 1: Hypertrophic fault events

\begin{tabular}{llllll}
\hline Circuit & $\begin{array}{l}\text { Number of } \\
\text { hypertrophic } \\
\text { faults }\end{array}$ & $\left|E_{g}\right|$ & $\left|E_{f-g}\right|$ & $\left|E_{f}-E_{g}\right|$ & $\frac{\left|E_{f}-E_{g}\right|}{\left|E_{f-g}\right|}$ \\
\hline s382 & 19 & 73459 & 3757416 & 71298 & 0.02 \\
s444 & 19 & 73349 & 3270133 & 65341 & 0.02 \\
$\mathbf{s 5 2 6}$ & 27 & 36177 & 1523038 & 88767 & 0.06 \\
$\mathbf{s 1 4 8 8}$ & 7 & 255948 & 1396374 & 121877 & 0.09 \\
$\mathbf{s 3 5 9 3 2}$ & 10 & 751122 & 2971640 & 458875 & 0.15 \\
\hline
\end{tabular}

ation analysis in Section 4. In this Table, five ISCAS89 circuits with only hypertrophic faults are simulated with STG3 test vectors. The hypertrophic faults are identified as described previously. For each circuit, $\left|E_{f-g}\right|$ and $\mid E_{f}$ $-E_{g} \mid$ of all identified hypertrophic faults are listed in columns 4 and 5 , and their ratio is given in the last column. The ratio clearly shows that $\left|E_{f}-E_{g}\right|$ is far less than $\left|E_{f-g}\right|$, being less than $15 \%$ for all listed circuits.

From this key observation, an efficient simulation algorithm targeted for hypertrophic faults will be proposed in the following Section.

\section{HYHOPE}

\subsection{Algorithm}

The above observation suggests that the hypertrophic faults should be simulated in parallel with logic simulation. Based on the key observation, the reduction of gate evaluations for hypertrophic faults is achieved in three ways:

(a) Reduce the simulation loads of the hypertrophic fault by simulating only the difference between two consecutive time frames of a faulty circuit, instead of simulating its huge differences from the good circuit. (b) Combine the simulation of hypertrophic faults with logic simulation.

(c) In addition, simulate the hypertrophic faults in parallel.

For non-hypertrophic faults, algorithms such as ROOFS-based algorithms are preferred, as these faults are only slightly different from the good circuit. Therefore the HyHOPE algorithm is as shown in Fig. 1.

All faults are in the regular fault list $F L$;

For each test vector \{

simulate good circuit and hypertrophic faults in parallel

drop detected hypertrophic faults;

while (there are faults $\in F L$ not simulated for this vector) \{

simulate fault(s) with the ROOFS-based algorithm

identify hypertrophic faults;

for each identified hypertrophic fault

mark the fault as hypertrophic fault \} remove it from $F L$

?

Fig. 1 HyHOPE algorithm

This simulation algorithm is essentially to simulate hypertrophic faults, as in the classical parallel-fault simulation [16], in which all faults are grouped into packets, and each packet of faults is simulated in parallel with the good circuit. The classical parallel fault simulation is not efficient, because most faults are non-hypertrophic and produce a difference from the good circuit smaller than that from the faulty circuits in the previous time frame. On the other hand, only the hypertrophic faults are in parallel simulated with the good circuit in HyHOPE. The key concept of the HyHOPE algorithm is that, by identifying the hypertrophic faults, we are able to simulate both kinds of fault, hypertrophic and non-hypertrophic, in the most efficient way.

\subsection{Implementation}

In this Section, the implementation of HyHOPE is given.

The proposed algorithm for simulating hypertrophic faults can be incorporated with existent fault simulators, such as ROOFS, and its parallel-fault versions, PROOFS and HOPE. We developed our simulator HyHOPE upon HOPE because the latter has the best performance and, most importantly, its source has been made publicly available by its authors.

In HyHOPE, procedures for hypertrophic fault identification and processing are built upon the original simulation mechanism of HOPE. Therefore the proven techniques of HOPE, such as using single-fault propagation to screen out single event faults with a short propagation path and using parallel-fault simulation to simulate multiple event faults and stem faults, are retained in HyHOPE for non-hypertrophic faults. All faults in the regular fault list are initially classified as non-hypertrophic. After a packet of faults has been simulated, the hypertrophic fault identification procedure is invoked to identify hypertrophic faults. The identified faults are then removed from the regular fault list into a hypertrophic fault list. At the next time frame, the faults in the hypertrophic fault list are simulated in parallel with the logic simulation. The original procedure for logic simulation is enhanced such that the good circuit and faulty circuits of hypertrophic faults can be simulated simultaneously. A fault in the hypertrophic fault list remains in the list until the fault is detected and dropped.

Accordingly, some data structures in HOPE are also modified. In HyHOPE, an extra word pair for each gate 
is added to record in parallel the good-circuit status and the hypertrophic faulty-circuit status. This word pair is evaluated during the parallel simulation of good and hypertrophic faulty circuits. The first bit of the word pair is used to represent the good-circuit status. After the parallel simulation, its value is copied to original computer words repesenting the good-circuit status. The original good-circuit words are used for both parallelfault simulation and single-fault propagation of nonhypertrophic faults, as in HOPE. It is possible to remove the original good-circuit words as, in HyHOPE, there is already a bit pair representing the good-circuit status. However, the good-circuit status then has to be retrieved during non-hypertrophic fault simulation and more overhead will be introduced in each gate evaluation. As the number of logic events is generally less than the number of fault events in sequential circuit fault simulation, we eliminate this overhead with a small increase in memory space.

In the present implementation of HyHOPE, the maximum number of hypertrophic faults to be simulated in parallel with logic simulation is limited by the given computer word length: 32 minus one for good-circuit simulation in our case. The hypertrophic fault identification procedure will not be invoked by HyHOPE when the number of currently simulated hypertrophic faults is equal to 31 . The number limitation does not affect the performance of HyHOPE in the evaluation, as the number of hypertrophic faults is small in general, and the space is reused when some identified hypertrophic faults are detected. We would also like to point out that the hypertrophic fault packet size can be extended to more than one word, and many hypertrophic faults can be simulated simultaneously when more than 31 hypertrophic faults are identified.

\section{Gate evaluation analysis}

In this Section, the efficiency of various fault simulation algorithms will be discussed, based on analysis of the cases for which gate evaluation must be performed.

In event-driven simulation, a gate evaluation may occur when a value transition of a gate input between two consecutive time frames or a value difference between good and faulty circuits takes place. Table 2 shows all the cases for changing status at a gate input between two consecutive time frames $T_{i-1}$ and $T_{i}$, for a fault. These cases are enumerated according to the four following conditions: (a) the faulty-circuit status $F_{i-1}$ is different from the good-circuit status $G_{i-1}$ at $T_{i-1}$,

(b) the good-circuit status is changed from $T_{i-1}$ to $T_{i}$,

(c) the faulty-circuit status is changed from $T_{i-1}$ to $T_{i}$,

(d) the faulty-circuit status $F_{i}$ is different from the good-circuit status $G_{i}$ at $T_{i}$.

A datum of value 1 in Table 2 indicates that a gate input meets the condition; otherwise, the value 0 is given. It is easy to see that four of the cases are not possible, and $\mathrm{C}_{0}, \mathrm{C}_{6}$ and $\mathrm{C}_{9}$ result in no gate evaluation in a worthy fault simulator.

For a given simulation algorithm, a case may or may not result in gate evaluation, and these characteristics determine the efficiency of the simulator. The simulation algorithms of ROOFS, concurrent-fault simulation and HyHOPE for hypertrophic faults are considered in this Section. Note that the discussion on ROOFS can also be applicable to its various parallelised versions, PROOFS and HOPE, because the parallelisation of faults can reduce but not completely eliminate the gate evaluations in the cases in Table 2.

In Fig. 2, the cases for which a simulation algorithm must perform gate evaluation are shown in the shaded area of the gate evaluation map. For example, a gate is evaluated in ROOFS for a fault if the gate has a gate input that falls into any of the cases $C_{3}, C_{5}, C_{7}, C_{9}, C_{11}$, $C_{13}$ and $C_{15}$. In concurrent-fault simulation, gate evaluation is performed for a fault if any of the cases $\mathrm{C}_{3}, \mathrm{C}_{5}$, $\mathrm{C}_{7}, \mathrm{C}_{10}, \mathrm{C}_{11}, \mathrm{C}_{12}, \mathrm{C}_{13}, \mathrm{C}_{14}, \mathrm{C}_{15}$, and $\mathrm{C}_{6-9}$ occurs, where $C_{6 \rightarrow 9}$ is the case where a gate has inputs that satisfy both conditions of $\mathrm{C}_{6}$ and $\mathrm{C}_{9}$. This is the case that a fault is in the fault list of the gate, and there are also logic events at gate inputs.

For both ROOFS and concurrent-fault simulation, these cases, indicated in their respective maps, are associated with gate evaluations independent of those of logic simulation, i.e. the gate evaluations of faults resulting from these cases must be performed in addition to those of Fig. 2a. On the other hand, for the hypertrophic faults in HyHOPE, they are simulated in parallel with logic simulation, and, hence, some of their gate evaluation cases coincide with those of logic simulation, as indicated in Fig. $2 d$. Therefore only the non-coincident cases, $\mathrm{C}_{3}$, $C_{10}$, and $C_{11}$, result in additional gate evaluations. As a result, it can be concluded that HyHOPE is superior to concurrent-fault simulation in dealing with hypertrophic faults, because the shaded area of the latter covers more than $C_{3}, C_{10}$, and $C_{11}$ and thus needs more gate evaluations than HyHOPE.

Table 2: Cases of gate evaluation

\begin{tabular}{llllll}
\hline Case & \multicolumn{5}{c}{ Gate input status } \\
\cline { 2 - 6 } & $F_{i-1} \neq G_{i-1}$ & $G_{i-1} \neq G_{i}$ & $F_{i-1} \neq F_{i}$ & $F_{i} \neq G_{i}$ & Examples \\
\hline$C_{0}$ & 0 & 0 & 0 & 0 & $0, x$ \\
$C_{1}$ & 0 & 0 & 0 & 1 & impossible \\
$C_{2}$ & 0 & 0 & 1 & 0 & impossible \\
$C_{3}$ & 0 & 0 & 1 & 1 & $1 \rightarrow 1 / 0, x \rightarrow x / 0$ \\
$C_{4}$ & 0 & 1 & 0 & 0 & impossible \\
$C_{5}$ & 0 & 1 & 0 & 1 & $1 \rightarrow 0 / 1, x \rightarrow 0 / x$ \\
$C_{6}$ & 0 & 1 & 1 & 0 & $0 \rightarrow 1,1 \rightarrow 0$ \\
$C_{7}$ & 0 & 1 & 1 & 1 & $x \rightarrow 1 / 0,0 \rightarrow 1 / x$ \\
$C_{8}$ & 1 & 0 & 0 & 0 & impossible \\
$C_{9}$ & 1 & 0 & 0 & 1 & $1 / 0,1 / x$ \\
$C_{10}$ & 1 & 0 & 1 & 0 & $1 / 0 \rightarrow 1,1 / x \rightarrow 1$ \\
$C_{11}$ & 1 & 0 & 1 & 1 & $1 / x \rightarrow 1 / 0,1 / 0 \rightarrow 1 / x$ \\
$C_{12}$ & 1 & 1 & 0 & 0 & $1 / 0 \rightarrow 0,1 / x \rightarrow x$ \\
$C_{13}$ & 1 & 1 & 0 & 1 & $1 / x \rightarrow 0 / x, x / 0 \rightarrow 1 / 0$ \\
$C_{14}$ & 1 & 1 & 1 & 0 & $1 / 0 \rightarrow x, 1 / x \rightarrow 0$ \\
$C_{15}$ & 1 & 1 & 1 & 1 & $1 / 0 \rightarrow 0 / 1,1 / x \rightarrow 0 / 1$ \\
\hline
\end{tabular}


The gate evaluation maps of ROOFS and HyHOPE show that, for hypertrophic faults, ROOFS has extra gate evaluations for $\mathrm{C}_{5}, \mathrm{C}_{7}, \mathrm{C}_{9}, \mathrm{C}_{13}$ and $\mathrm{C}_{15}$, compared with
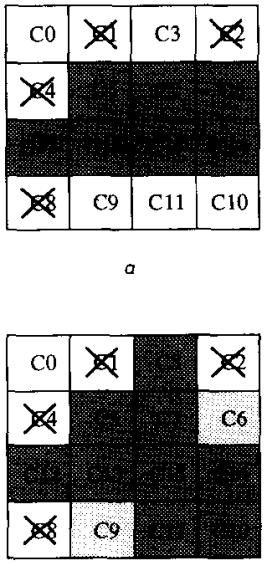

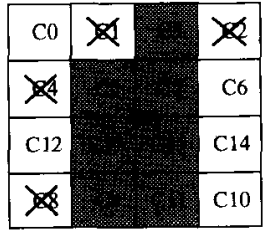

$b$

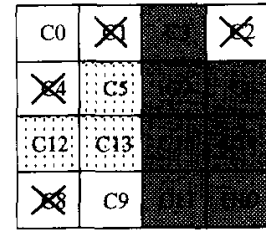

Fig. 2 Gate evaluation maps for various fault simulation algorithms a Logic simulation

$b$ ROOFS (ail faults)

c Concurrent-fault simulation (all faults)

d HyHOPE (hypertrophic faults)

- The darkly shaded area indicates the cases for which a simulation algorithm must perform gate evaluations

For concurrent-fault simulation, in addition to the dark shaded area, gate evaluation is required for the case $\mathrm{C}_{6-9}$ indicated by the lightly shaded area in $c$ 闬 Simulation for hypertrophic faults in HyHOPE is combined with logic simulation, as indicated by the dotted area in $d$

HyHOPE, whereas HyHOPE has an extra evaluation for $\mathrm{C}_{10}$ compared with HOPE. From Table 2 , it can be seen that $\mathrm{C}_{10}$ occurs when the faulty circuit changes status while the good circuit remains unchanged. For hypertrophic faults, such an occurrence is far less likely than cases such as $C_{5}, C_{9}$ and $C_{13}$, for which the faulty circuit is plagued by $X s$ and the good circuit has known status. As a result, HyHOPE can achieve better efficiency than ROOFS for hypertrophic faults. As HyHOPE adopts the same algorithm as ROOFS for non-hypertrophic faults, the overall performance of HyHOPE is significantly better than ROOFS-based simulators, as will be demonstrated experimentally in the following Section.

It is also interesting to compare ROOFS and concurrent-fault simulation in terms of gate evaluation maps. For lightly active faults, ROOFS is superior to the concurrent-fault simulation, because the restoration, $C_{10}$ and $C_{12}$, is not required in ROOFS. As most faulty circuits are only slightly different from the good circuit, it can be concluded that ROOFS is more efficient than the concurrent-fault simulation. The conclusion is clearly demonstrated by the results in References 2 and 3, in which ROOFS had much fewer events than an existent concurrent-fault simulator CSIM [15] for all evaluated circuits. However, for hypertrophic faults, the area of $\left\{C_{5}, C_{9}, C_{12}, C_{13}\right\}$ is likely to be larger than others, as most of the faulty-circuit statuses of a hypertrophic fault remain unknown. Notably, the size of $\mathrm{C}_{9}$ is conceivably large for a hypertrophic fault. Thus it would degrade the performance of ROOFS, and concurrent-fault simulation, although not as efficient as HyHOPE, might be more effective than ROOFS for hypertrophic faults, although there have not yet been any published results detailed enough to support this deduction.

The above analysis indicates that the simulation algorithm for hypertrophic fault in HyHOPE is superior to the concurrent-fault simulation and ROOFS. In the following Section, experimental results will be given to show that HyHOPE indeed has better performance than HOPE, a ROOFS-based simulator.

\section{$5 \quad$ Experimental results}

Our fault simulator HyHOPE was evaluated on ISCAS89 benchmark circuits [21] with test vectors generated by STG3 $[18,19]$ and a simulation-based ATPG [19]. First, we conducted the experiment with STG3 test vectors. Table 3 shows a summary of these circuits and

\begin{tabular}{|c|c|c|c|c|c|}
\hline \multirow[t]{3}{*}{ Circuit } & \multirow{3}{*}{$\begin{array}{l}\text { Number } \\
\text { of } \\
\text { vectors }\end{array}$} & \multirow{3}{*}{$\begin{array}{l}\text { Number } \\
\text { of } \\
\text { faults }\end{array}$} & \multirow{3}{*}{$\begin{array}{l}\text { Number } \\
\text { of } \\
\text { hypertrophic } \\
\text { faults }\end{array}$} & \multicolumn{2}{|c|}{ Fault coverage, $\%$} \\
\hline & & & & \multirow{2}{*}{$\begin{array}{l}\text { sure } \\
\text { HOPE } \\
\text { HyHOPE }\end{array}$} & \multirow{2}{*}{$\begin{array}{l}\text { potential } \\
\text { PTD }\end{array}$} \\
\hline & & & & & \\
\hline s208 & 111 & 215 & 24 & 63.72 & 72.09 \\
\hline s298 & 162 & 308 & 15 & 85.71 & 88.64 \\
\hline s344 & 91 & 342 & 29 & 96.20 & 97.95 \\
\hline s382 & 2463 & 399 & 19 & 90.98 & 94.74 \\
\hline s400 & 1282 & 424 & 19 & 82.78 & 86.56 \\
\hline$\$ 420$ & 173 & 430 & 37 & 41.63 & 50.70 \\
\hline s444 & 1881 & 474 & 19 & 89.45 & 92.62 \\
\hline s526 & 754 & 555 & 27 & 75.32 & 78.38 \\
\hline s641 & 133 & 467 & 12 & 86.30 & 87.79 \\
\hline s713 & 107 & 581 & 37 & 80.90 & 83.13 \\
\hline $\mathbf{s} 820$ & 411 & 850 & 5 & 81.88 & 82.12 \\
\hline s832 & 377 & 870 & 5 & 81.38 & 81.49 \\
\hline$s 838$ & 137 & 857 & 33 & 29.64 & 38.39 \\
\hline s953 & 16 & 1079 & 31 & 7.79 & 15.01 \\
\hline s1238 & 349 & 1355 & 2 & 94.69 & 94.69 \\
\hline s1423 & 36 & 1515 & 51 & 24.42 & 28.12 \\
\hline s1488 & 590 & 1486 & 7 & 92.60 & 92.80 \\
\hline s1494 & 469 & 1506 & 7 & 91.10 & 91.43 \\
\hline s5378 & 408 & 4603 & 38 & 74.02 & 75.32 \\
\hline s35932 & 86 & 39094 & 10 & 87.99 & 88.04 \\
\hline
\end{tabular}

test vectors. Throughout all the experiments, the initial states of all the flip-flops were assumed to be unknown $X$ s. The performance of HyHOPE was compared with those of HOPE and PTD, which is an option of HOPE to drop potentially detected faults.

Table 3 also shows the fault coverages of exact fault simulation, such as HyHOPE and HOPE, and the approximate fault simulation by PTD. As expected, PTD always gives an optimistic result. The difference can be significant, and more than $8 \%$ difference in fault coverage was observed for $s 208, s 420$, and $s 838$. The number of hypertrophic faults identified and processed by HyHOPE is also given. For some circuits, this number is larger than 31 , which means that, during the simulation, some identified hypertrophic faults were detected and their bit space was reused in the following time frames. It can also be seen that the hypertrophic faults are generally less than $5 \%$ of the total faults. In particular, the identified hypertrophic faults in $\mathrm{s} 35932$ are only $0.03 \%$, yet almost half the fault gate evaluations were due to this small number of faults.

The run times of HyHOPE and HOPE on a SUN Sparc 2 workstation are reported in Table 4 . The performance of HyHOPE can be clearly seen in this Table. The average speed-up ratio of HyHOPE over HOPE is 1.65. As shown in Table 4, HyHOPE is faster than HOPE for all but one circuit. For circuit s1238, HyHOPE is slightly slower than HOPE, because the circuit has no feedback loop, and there are very few 
serious hypertrophic faults. The overhead for hypertrophic fault identification and processing makes the performance of HyHOPE slightly slower than HOPE. It is

Table 4: CPU times for STG3 test vectors

\begin{tabular}{lrrrrrr}
\hline Circuit & \multicolumn{3}{c}{ CPU time, } & & \multicolumn{2}{c}{ Speed-up over HOPE } \\
\cline { 2 - 4 } \cline { 5 - 6 } & HOPE & PTD & HyHOPE & PTD & HyHOPE \\
\hline s208 & 0.33 & 0.20 & 0.25 & & 1.65 & 1.32 \\
s298 & 0.50 & 0.27 & 0.28 & & 1.82 & 1.79 \\
s344 & 0.35 & 0.22 & 0.25 & & 1.59 & 1.40 \\
s382 & 9.22 & 2.12 & 2.22 & & 4.35 & 4.15 \\
s400 & 6.15 & 2.08 & 2.27 & & 2.96 & 2.71 \\
s420 & 1.18 & 0.63 & 0.78 & & 1.87 & 1.51 \\
s444 & 10.12 & 3.67 & 3.77 & & 2.76 & 2.68 \\
s526 & 5.92 & 3.08 & 3.18 & & 1.92 & 1.86 \\
s641 & 0.60 & 0.42 & 0.52 & & 1.43 & 1.15 \\
s713 & 0.63 & 0.48 & 0.55 & & 1.32 & 1.15 \\
s820 & 2.18 & 1.47 & 1.63 & & 1.48 & 1.34 \\
s832 & 2.10 & 1.42 & 1.58 & & 1.48 & 1.33 \\
s838 & 2.93 & 1.70 & 2.18 & & 1.72 & 1.34 \\
s953 & 0.83 & 0.80 & 0.83 & & 1.03 & 1.00 \\
s1238 & 2.00 & 2.01 & 2.12 & & 1.00 & 0.94 \\
s1423 & 2.02 & 1.72 & 1.90 & & 1.17 & 1.06 \\
s1488 & 6.63 & 3.60 & 3.93 & & 1.84 & 1.69 \\
s1494 & 5.35 & 2.93 & 3.45 & & 1.83 & 1.55 \\
s5378 & 26.73 & 14.23 & 17.88 & & 1.87 & 1.49 \\
s35932 & 94.90 & 60.93 & 64.68 & & 1.83 & 1.47 \\
Average & speed-up ratio & & 1.83 & 1.65 \\
\hline
\end{tabular}

aso interesting to note that, for circuits $s 382, s 400$ and $\mathrm{s} 444$, the speed-up ratios over HOPE are more than 2 , and their identified hypertrophic faults are less than 5\% of total faults. This is because, as discussed in Section 2 the hypertrophic faults, although few in number, produce a large number of unknown values that are different from the good-circuit values. Many of these faults can only be potentially detected. For instance, none of the identified hypertrophic faults in s35932 can be surely detected by the given test sequence. As a result, fault simulators, such as ROOFS and HOPE, that process the differences between faulty circuits and good circuits require a great number of gate evaluations for these faults. In HyHOPE, simulating these hypertrophic faults in parallel with the good circuit leads to a significant improvement.
For evaluation purposes, we also show the run time of the approximate fault simulator PTD in Table 4. As PTD drops the potentially detected faults, which are mostly hypertrophic faults, its performance can be used as a goal that an exact fault simulator of the same basic simulation mechanism strives to achieve. As expected PTD outdoes its exact version HOPE by $83 \%$ on average, with some sacrifice in accuracy. When compared with HyHOPE, also based on HOPE, PTD is only slightly faster than the exact simulator HyHOPE. This indicates that HyHOPE not only preserves the accuracy but also has the performance approaching that of the approximate simulator

To examine our proposed simulation algorithm for hypertrophic faults in detail, the number of gate evaluations for faulty-circuit simulation of HOPE, PTD and HyHOPE are listed in Table 5. For HOPE and PTD, the fault gate evaluations consist of single-fault propagation candidacy test and parallel-fault simulation. The listed gate evaluations of HyHOPE consist of two parts: the column 'fault' in HyHOPE is the number of fault gate evaluations generated by non-hypertrophic faults that are simulated as in HOPE; and the column 'extra' lists the extra evaluations for simulating hypertrophic faults in parallel with logic simulation, as proposed in our simulation algorithm. Therefore the number of total fault gate evaluations with HyHOPE is the sum of these two numbers. From Table 5, it can be seen that more than $40 \%$ of fault gate evaluations in HOPE are reduced by HyHOPE on average, which is approaching the ratio achieved by PTD. Furthermore, there are many circuits, such as s382, s444, s526, s832, s1488 and s35932, where the efficiency of HyHOPE is very close to PTD.

A new version of HOPE, HOPE1.1 [12] incorporates three additional heuristics: functional injection; static ordering by fanout free region; and dynamic fault ordering of potentially detected faults. It appears that functional injection is the most effective of the three and is able to reduce fault site events. However, the overall improvement is not significant: $12 \%$, except for two circuits, s5378 and s35932. The improvement of these two circuits mostly comes from functional injection, which is not implemented in HyHOPE. Even so, HyHOPE still has

Table 5: Number of gate evaluations for STG3 test vectors

\begin{tabular}{|c|c|c|c|c|c|c|c|}
\hline \multirow[t]{3}{*}{ Circuit } & \multicolumn{5}{|c|}{ Number of gate evaluations for faults } & \multicolumn{2}{|c|}{ Evaluation ratio over HOPE } \\
\hline & \multirow[t]{2}{*}{ HOPE } & \multirow[t]{2}{*}{ PTD } & \multicolumn{3}{|c|}{ HyHOPE } & \multirow[t]{2}{*}{ PTD } & \multirow[t]{2}{*}{ HyHOPE } \\
\hline & & & fault & extra & total & & \\
\hline $\begin{array}{l}s 208 \\
s 298 \\
s 344 \\
s 382 \\
s 400 \\
s 420 \\
s 444 \\
s 526 \\
s 641 \\
s 713 \\
s 820 \\
s 832 \\
s 838 \\
s 953 \\
s 1238 \\
s 1423 \\
s 1488 \\
s 1494 \\
s 5378 \\
s 35932\end{array}$ & $\begin{array}{r}21098 \\
30274 \\
22264 \\
610068 \\
434632 \\
108471 \\
712839 \\
397639 \\
36379 \\
38524 \\
187090 \\
175211 \\
298443 \\
53278 \\
117088 \\
128094 \\
487501 \\
405012 \\
2038037 \\
6149767\end{array}$ & $\begin{array}{r}9506 \\
10140 \\
6603 \\
109252 \\
117698 \\
58920 \\
228080 \\
202697 \\
14605 \\
17768 \\
101708 \\
96420 \\
187776 \\
50003 \\
116352 \\
102125 \\
153150 \\
147095 \\
850656 \\
3424269\end{array}$ & $\begin{array}{r}9758 \\
9983 \\
6520 \\
105695 \\
116347 \\
64633 \\
219532 \\
197856 \\
14660 \\
18532 \\
102782 \\
97029 \\
230462 \\
51967 \\
117106 \\
108515 \\
153486 \\
147626 \\
1025816 \\
3557019\end{array}$ & $\begin{array}{r}4572 \\
3627 \\
4681 \\
22767 \\
16644 \\
15103 \\
19595 \\
25673 \\
4627 \\
5422 \\
11867 \\
10444 \\
23910 \\
294 \\
63 \\
5251 \\
41216 \\
34201 \\
110493 \\
228301\end{array}$ & $\begin{array}{r}14330 \\
13610 \\
11201 \\
128462 \\
132991 \\
79736 \\
239127 \\
223529 \\
19087 \\
23954 \\
114649 \\
107473 \\
254372 \\
52261 \\
117169 \\
113766 \\
194702 \\
181827 \\
1136309 \\
3785320\end{array}$ & $\begin{array}{l}0.451 \\
0.335 \\
0.297 \\
0.179 \\
0.271 \\
0.542 \\
0.320 \\
0.510 \\
0.401 \\
0.461 \\
0.544 \\
0.550 \\
0.629 \\
0.939 \\
0.994 \\
0.797 \\
0.314 \\
0.363 \\
0.417 \\
0.557\end{array}$ & $\begin{array}{l}0.679 \\
0.450 \\
0.503 \\
0.211 \\
0.306 \\
0.735 \\
0.335 \\
0.562 \\
0.525 \\
0.622 \\
0.613 \\
0.613 \\
0.852 \\
0.981 \\
1.001 \\
0.888 \\
0.399 \\
0.449 \\
0.558 \\
0.616\end{array}$ \\
\hline \multicolumn{6}{|c|}{ Average evaluation ratio } & 0.494 & 0.595 \\
\hline
\end{tabular}


fewer fault gate evaluations than HOPE1.1 for these two circuits, and the average speed-up for all circuits is $47 \%$ over HOPE1.1.

We also conducted the experiment with the test set generated by a simulation-based ATPG [19] (see Table 6). Although the general quality of this test set is comparable with that of STG3, for individual circuits, the fault coverage shows significant difference. However, HyHOPE still outperforms HOPE by an average speedup of 1.90 . It can be seen that, for most circuits, the number of identified hypetrophic faults is similar to that of STG3. The performance of HyHOPE is close to that of PTD, except for the circuit $\mathbf{s 1 4 2 3}$. The discrepancy comes from the fact that many serious hypertrophic faults in the circuit are activated by this test sequence. The current implementation of HyHOPE can only simulate 31 hypertrophic faults in parallel. As a result, a few serious hypertrophic faults are simulated with nonhypertrophic fault simulation instead of the proposed efficient algorithm. For this case, the performance of HyHOPE can be further improved by extending the packet size for hypertrophic fault simulation, as described in Section 3.2. However, for most circuits in our experiment, the extension of packet size was not needed. From the above Tables, it is clear that the efficient hypertrophic fault simulation in HyHOPE leads to a significant improvement for both test sets generated by STG3 and the simulation-based ATPG.

A fair comparison of HyHOPE with other well-known ROOFS-based fault simulators, such as PARIS [7] and PROOFS $[3,4]$, can only be indirect, owing to the difference in the machines used and in implementation details. For PROOFS, fortunately, it has been reported in Reference 5 that HOPE had a speed-up advantage of 2 . Therefore we estimate that HyHOPE could have a speed-up ratio of more than 3 over PROOFS, as we built HyHOPE on HOPE. In the case of PARIS, which exploits pattern parallelism, we compare HyHOPE with a simulator implemented according to the description in Reference 7 of PARIS*. The run time result with the STG3 test set is listed in Table 7. The diversified comparison results reflect the different natures of parallel fault in HyHOPE and parallel pattern in PARIS*. For

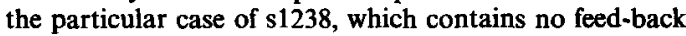

loop, PARIS* behaves as PPSFP [9] and is very efficient. However, for some circuits, such as s298, s382, s444, s526, s1494, and s35932, PARIS* is clearly outperformed by HyHOPE. For some of these circuits, Table 7: Comparison for STG3 test vectors

\begin{tabular}{|c|c|c|c|}
\hline \multirow[t]{2}{*}{ Circuit } & \multicolumn{2}{|c|}{ CPU time } & \multirow{2}{*}{$\frac{\text { Time ratio }}{\text { PARIS } * / \text { HyHOPE }}$} \\
\hline & PARIS* & HyHOPE & \\
\hline s208 & 0.22 & 0.25 & 0.880 \\
\hline s298 & 0.82 & 0.28 & 2.929 \\
\hline$s 344$ & 0.68 & 0.25 & 2.720 \\
\hline$\$ 382$ & 7.37 & 2.22 & 3.320 \\
\hline$\$ 400$ & 7.40 & 2.27 & 3.260 \\
\hline$\$ 420$ & 0.65 & 0.78 & 0.833 \\
\hline$\$ 444$ & 15.53 & 3.77 & 4.119 \\
\hline s526 & 12.05 & 3.18 & 3.789 \\
\hline s641 & 0.60 & 0.52 & 1.154 \\
\hline$s 713$ & 0.75 & 0.55 & 1.364 \\
\hline 5820 & 2.73 & 1.63 & 1.675 \\
\hline s832 & 3.27 & 1.58 & 2.070 \\
\hline 5838 & 1.43 & 2.18 & 0.656 \\
\hline s953 & 0.37 & 0.83 & 0.446 \\
\hline $\mathbf{s} 1238$ & 0.60 & 2.12 & 0.283 \\
\hline s1423 & 4.03 & 1.90 & 2.121 \\
\hline s1488 & 13.10 & 3.93 & 3.333 \\
\hline$s 1494$ & 12.95 & 3.45 & 3.754 \\
\hline $\begin{array}{l}\mathbf{s} 5378 \\
\mathbf{3 5 9 3 2}\end{array}$ & $\begin{array}{r}10.88 \\
1720.20\end{array}$ & $\begin{array}{l}17.88 \\
64.68\end{array}$ & $\begin{array}{r}0.609 \\
26.596\end{array}$ \\
\hline
\end{tabular}

PARIS* is even inferior to its single-pattern version. The last observation has also been reported in References ? and 8. Based on our comparison with PARIS*, it appears that HyHOPE delivers a consistently better performance. However, the comparative merits of parallel-fault simulation and parallel-pattern simulation deserve more thorough investigation.

In summary, HyHOPE improves the performance of HOPE by about $65 \%$. Furthermore, the experiment shows that, with our efficient algorithm for simulating hypertrophic faults, the exact fault simulator can be almost as fast as the approximate one, without any sacrifice in accuracy.

\section{Conclusions}

The efficiency of modern fault simulation algorithms depends largely on the degree of difference between the

Table 6: Results for simulation-based ATPG test vectors

\begin{tabular}{|c|c|c|c|c|c|c|c|c|c|}
\hline \multirow[t]{2}{*}{ Circuit } & \multirow[t]{2}{*}{$\begin{array}{l}\text { Number } \\
\text { of vectors }\end{array}$} & \multirow{2}{*}{$\begin{array}{l}\text { Number of } \\
\text { hypertrophic } \\
\text { faults }\end{array}$} & \multicolumn{2}{|c|}{$\begin{array}{c}\text { Fault } \\
\text { coverage } \%\end{array}$} & \multicolumn{3}{|c|}{$\begin{array}{c}\text { CPU time, } \\
s\end{array}$} & \multicolumn{2}{|c|}{$\begin{array}{l}\text { Speed-up } \\
\text { over HOPE }\end{array}$} \\
\hline & & & sure & potential & HOPE & PTD & HYHOPE & PTD & HyHOPE \\
\hline $\begin{array}{l}s 208 \\
s 298 \\
s 344 \\
s 382 \\
s 400 \\
s 420 \\
s 444 \\
s 526 \\
s 641 \\
s 713 \\
s 820 \\
s 832 \\
s 838 \\
s 953 \\
s 1238 \\
s 1423 \\
s 1488 \\
s 1494 \\
s 5378 \\
s 35932\end{array}$ & $\begin{array}{r}400 \\
350 \\
300 \\
1014 \\
1512 \\
200 \\
1692 \\
2682 \\
211 \\
350 \\
900 \\
703 \\
150 \\
156 \\
500 \\
1451 \\
300 \\
300 \\
501 \\
298\end{array}$ & $\begin{array}{r}22 \\
15 \\
28 \\
19 \\
17 \\
41 \\
19 \\
27 \\
29 \\
17 \\
3 \\
3 \\
38 \\
31 \\
3 \\
41 \\
5 \\
5 \\
49 \\
10\end{array}$ & $\begin{array}{r}63.26 \\
85.71 \\
95.03 \\
88.97 \\
88.21 \\
40.70 \\
85.87 \\
79.28 \\
84.37 \\
81.76 \\
93.88 \\
91.61 \\
29.06 \\
8.34 \\
91.44 \\
82.18 \\
94.01 \\
93.29 \\
69.15 \\
88.26\end{array}$ & $\begin{array}{l}70.70 \\
88.67 \\
96.78 \\
93.23 \\
91.74 \\
48.84 \\
90.93 \\
82.34 \\
86.72 \\
83.13 \\
94.00 \\
91.84 \\
37.57 \\
20.85 \\
91.44 \\
86.14 \\
94.35 \\
93.63 \\
70.61 \\
88.28\end{array}$ & $\begin{array}{r}0.77 \\
0.88 \\
0.78 \\
4.07 \\
5.82 \\
1.28 \\
7.53 \\
13.28 \\
0.92 \\
1.45 \\
3.92 \\
3.22 \\
3.52 \\
6.13 \\
3.15 \\
58.37 \\
3.58 \\
3.43 \\
31.13 \\
171.80\end{array}$ & $\begin{array}{r}0.35 \\
0.40 \\
0.33 \\
1.05 \\
1.28 \\
0.67 \\
1.82 \\
3.15 \\
0.52 \\
0.85 \\
2.45 \\
2.10 \\
2.10 \\
5.17 \\
3.15 \\
17.00 \\
2.22 \\
2.10 \\
16.10 \\
91.00\end{array}$ & $\begin{array}{r}0.43 \\
0.47 \\
0.42 \\
1.22 \\
1.60 \\
0.83 \\
2.13 \\
3.77 \\
0.62 \\
1.03 \\
2.73 \\
2.22 \\
2.63 \\
5.83 \\
3.25 \\
35.77 \\
2.33 \\
2.35 \\
21.13 \\
97.72\end{array}$ & $\begin{array}{l}2.20 \\
2.20 \\
2.42 \\
3.88 \\
4.55 \\
1.91 \\
4.14 \\
4.22 \\
1.77 \\
1.71 \\
1.60 \\
1.53 \\
1.68 \\
1.19 \\
1.00 \\
3.43 \\
1.61 \\
1.63 \\
1.93 \\
1.89\end{array}$ & $\begin{array}{l}1.79 \\
1.87 \\
1.86 \\
3.34 \\
3.64 \\
1.54 \\
3.54 \\
3.52 \\
1.48 \\
1.41 \\
1.44 \\
1.45 \\
1.34 \\
1.05 \\
0.97 \\
1.63 \\
1.54 \\
1.46 \\
1.47 \\
1.76\end{array}$ \\
\hline \multicolumn{8}{|c|}{ Average speed-up ratio } & 2.32 & 1.90 \\
\hline
\end{tabular}


good circuit and its faulty versions, i.e. the number of fault effects. For most faults, the fault effects are few in number, and these simulators attain high efficiency by evaluating only a small number of gates for each fault. However, a small number of hypertrophic faults in a circuit may produce a great number of $X \mathrm{~s}$, which are evidently different from good values. As a result, a simulator taking advantage of such a difference ends up heavily loaded with a large number of gate evaluations for the hypertrophic faults, which may account for as much as half the CPU time.

In this paper, we have proposed a novel and exact fault simulation algorithm to identify hypertrophic faults during the fault simulation and efficiently to simulate hypertrophic faults for sequential circuits such that their effect on performance is minimised. The reduction of hypertrophic fault events by the proposed simulation algorithm is threefold:

(a) The algorithm simulates only those hypertrophic fault events resulting from a difference with the previous time frame rather than with the good circuit.

(b) Each hypertrophic fault is simulated in parallel with logic simulation. The events can be significantly reduced by $(a)$ and $(b)$, as stated in the key observation.

(c) In addition, these hypertrophic faults are simulated in parallel for even further reduction.

Based on this algorithm, a fault simulator HyHOPE for efficient and exact hypertrophic fault simulation has been implemented upon HOPE. The experimental results have shown that HyHOPE reduces by about $40 \%$ the fault gate evaluations of HOPE, and the average speed-up ratios over HOPE for STG3 test vectors and simulationbased ATPG test vectors are 1.65 and 1.90 , respectively. Furthermore, the results have also indicated that the performance of HyHOPE is close to the approximate simulator without any sacrifice in accuracy. The proposed HyHOPE algorithm is targeted at the single stuck-atfault model. We would like to point out that the proposed heuristics for hypertrophic fault simulation can be easily extended to fault simulation targeted at other fault models where hypertrophic fault effect may occur, such as transition fault $[22,23]$ and stuck-open fault [24].

\section{References}

1 ROTH, J.P., BOURICIUS, W.G., and SCHNEIDER, P.R.: 'Programmed algorithms to compute tests to detect and distinguish between failure in logic circuits', IEEE Trans., 1967, EC-16, (5), pp. $567-580$

2 CHENG, W.T., and YU, M.L.: 'Differential fault simulation - a fast, memory efficient sequential circuit-fault simulator'. Proc. 26th Design automation Conference, June 1989, pp. 424-428
3 CHENG, W.T., and PATEL, J.H.: 'PROOFS: a super fast fault simulator for sequential circuits'. Proc. European Conferenco on Design automation, March 1990, pp. 475-479

4 NIERMANN, T.M., CHENG, W.T., and PATEL, J.H.: 'PROOFS a fast, memory efficient sequential circuit fault simulator', IEEE Trans., 1992, CAD-11, (2), pp. 198-207

5 LEE, H.K., and HA, D.S.: 'HOPE: an efficient parallel fault simulator for synchronous sequential circuits'. Proc. 29th Design automation Conference, June 1992, pp. 336-340

6 KUNG, C.P., and LIN, C.S.: 'Parallel sequence fault simulation for synchronous sequential circuits'. Proc. European Conference on Design automation, March 1992, pp. 434-438

7 GOUDERS, N., and KAIBEL, R.: 'PARIS: a parallel pattern fault simulator for synchronous sequential circuits'. Proc. Int. Conf. on simulator for synchronous sequential circuits'.
Computer-aided design, Nov, 1991, pp. 542-545

8 MOJTAHEDI, M., and GEISSELHARDT, W.: New methods for parallel pattern fault simulation for synchronous sequential circuits' Proc. Int. Conf. on Computer-aided design, Nov. 1993, pp. 2-5

9 WAICUKAUSKI, J.A., EICHELBERGER, E.B., FORLENZA, D.O., LINDBLOOM, E., and MCCARTHY, T.: 'Fault simulation for structured VLSI', VLSI Syst. Des., Dec. 1985, pp. 20-32

10 GAI, S., MONTESSORO, P.L., and SOMENZI, F.: "The performance of the concurrent fault simulation algorithms in MOZART'. Proc. 25th Design automation Conference, June 1988 , pp. $682-697$.

11 GABODI, G., GAI, S., and SONZA REORDA, M.: 'Fast differential fault simulation by dynamic fault ordering'. Proc. International Conference on Computer design, 1991, pp. 60-63

12 LEE, H.K., and HA, D.S. : $\bar{C}^{-}$New methods of improving parallel fault simulation in synchronous sequential circuits'. Proc. Int. Conf. on Computer-aided design, Nov. 1993, pp. 10-17

13 ULRICH, E.G., and BAKER, T.: 'The concurrent fault simulation of nearly identical digital networks'. Proc. 10th Design automation Workshop, June 1973, 6, pp. 145-150

14 LEVENDEL, Y.H., and MENON, P.R.: 'Fault-simulation methods - extensions and comparison', Bell Syst. Tech. J., 1981, 60, (9), pp. 2235-2259

15 DAVIDSON, S., and LEWANDOWSKI, J.L.: 'ESIM/AFS - a concurrent architectural level fault simulator'. Int. Test Conf., 1985, pp. $663-698$

16 SESHU, S.: 'On an improved diagnosis program', IEEE Trans, 1965, EC-14, pp. 76-79

17 CHENG, W.-T.: 'SPLIT circuit model for test generation'. Proc 25th Design automation Conf., June 1988, pp. 96-101

18 CHENG, W.-T.: 'The BACK algorithm for sequential test generation'. Proc. Int. Conf. Computer design, Oct. 1988, pp. 66-69

19 PAN, S.S.: 'A directed search method for sequential test generation'. National Taiwan University M.S. Thesis, 1991

20 NIERMANN, T., and PATEL, J.H.: 'HITECH: a test generation package for sequential circuits'. Proc. European Conf. on Design automation, 1991, pp. 214-218

21 BRGLEZ, F., BRYAN, D., and KOZMINSKI, K.: 'Combinationa profiles of sequential circuits'. Proc. Int. Symposium on Circuits \& systems, May 1989, pp. 1929-1934

22 WAICUKAUSKI, R.L., LINDBLOOM, E., ROSE, B., and IYENGAR, V.: 'Transition fault simulation', IEEE Design \& Test, April 1987, pp. 32-38

23 CHENG, K.-T.: 'Transition fault simulation for sequential circuits'. Proc. Int. Test Conf., Nov. 1992, pp. 723-731

24 WADSACK, R.L.: 'Fault modeling and logic simulation of CMOS and NMOS integrated circuits', Bell Syst. Tech. J., May-June 1978, pp. $1449-1474$ 